\title{
De la fundación de un país al muro fronterizo: una perspectiva social e histórica de la migración en Estados Unidos de Norteamérica
}

Pablo Alejandro Berri ${ }^{1}$

\section{Contribución en la Sección Política Internacional}

Resumen: El discurso del actual presidente norteamericano, Donald Trump, que ha reivindicado la construcción de un muro en la frontera de ese país con México; ha manejado cifras que superaban la estimación existente de extranjeros no autorizados residiendo en el país y cuya disputa con el partido demócrata llevó al shutdown del gobierno más extenso de la historia del país, conducen a pensar que el tema de la inmigración ilegal en los Estados Unidos es creciente. Sin embargo, esa cifra no solamente no aumentó sino que descendió (Warren, 2016), en el marco de un contexto que puede dar cuenta de discriminación y barreras para la integración plena de los migrantes.

El texto considera brevemente el panorama migratorio en los Estados Unidos, proponiéndose revisar si las posturas anti-migratorias resultan novedosas en la historia del país, cuáles son los factores que condicionan los procesos migratorios a dicho país y cuáles sus características y si el despliegue de políticas adversas a los migrantes es patrimonio exclusivo del partido republicano.

Se concluye que las políticas que dificultan u obstaculizan la migración no son novedosas dentro de la historia estadounidense, señalándose cómo, desde la instancia fundacional del país hasta el presente, la relación con el migrante ha estado/está sesgada por una tendencia que aprovecha hábilmente las circunstancias, sean estas económicas (considerando al migrante como mano de obra barata), políticas (considerando a los migrantes como potenciales votantes) o relacionadas a la opinión pública (considerando al migrante como depositario de sentimientos negativos en justificación de una situación adversa).

\section{Introducción}

La prédica del presidente estadounidense, Donald Trump, en referencia a los procesos migratorios, en particular los de tipo irregular ${ }^{2}$, son de público conocimiento; uno de los pilares centrales de su campaña fue la tesis de que los problemas socioeconómicos de

\footnotetext{
${ }^{1}$ Estudiante avanzando de Psicología de la Facultad de Ciencias Sociales de la Universidad de Palermo, docente de Nivel Secundario y titular de Equipos de Distrito en la Modalidad de Psicología Comunitaria y Pedagogía Social de la Dirección General de Cultura y Educación de Buenos Aires.

2 Se comprende como irregular a todo aquel migrante que ingresa y permanece sin cumplir los requisitos legales del país destino (International Organization for Migration, 2010).
} 
los estadounidenses se debían a la inmigración y que un muro fronterizo remediaría dicho conflicto.

A su vez, y como ejemplo de la relevancia que cobra la cuestión migratoria en la actualidad estadounidense, podría destacarse el reciente, y más largo de la historia ${ }^{3}$, government shutdown; ${ }^{4}$ hecho que encuentra su punto central en una lucha presupuestaria entre republicanos y demócratas; Trump exigía un mayor presupuesto, para la construcción del muro fronterizo con los Estados Unidos de México, al que los demócratas se oponían.

Ahora bien, ante este panorama es válido reflexionar sobre algunas consideraciones de carácter social e histórico en torno a los Estados Unidos de Norteamérica. ¿Es exclusivo del partido republicano el despliegue de políticas adversas a los migrantes? ¿Las posturas anti-migratorias son novedosas en la historia estadounidense? ¿Qué factores son los que condicionan los procesos migratorios a dicho país y qué características tienen?

\section{Desarrollo}

Passel (2006) estima que de un total de 47 millones de migrantes, unos 11 millones residen en condición irregular. A su vez, varias investigaciones señalan que gran parte de la migración que reciben los Estados Unidos proviene de los Estados Unidos de México (Herrera Lima, 2012; Tuíran \& Avila, 2013), siendo unos 11.7 millones de mexicanos que residían, para el año 2010, en suelo norteamericano ${ }^{5}$. A su vez, existen diferentes investigaciones que sostienen que la tesis de que la migración de tipo irregular, se incrementó en los últimos años es falsa, destacando el hecho contrario, producto de un endurecimiento en las políticas migratorias a partir del atentando al World Trade Center (Warren, 2016).

La situación de los migrantes residentes en Estados Unidos es compleja. Por ejemplo, el número de hispanos que afirman que la discriminación es un problema se incrementó del 54\% al 61\% entre los años 2007 y 2010 (López, Morin \& Taylor, 2010). A su vez, los jóvenes migrantes encuentran como barrera, el uso del idioma y confrontaciones de tipo cultural, que impiden una integración plena (Adelma \& Taylor, 2015).

Además, la condición de los migrantes irregulares, principal baza de los críticos en contra de la migración, no es tan estable como se supondría ${ }^{6}$. Castles (2010) sostiene que

\footnotetext{
${ }^{3} 35$ días entre diciembre del 2018 y enero del 2019 (Zaveri, Gates \& Zraick, 2019).

4 Se conoce como government shutdown al proceso mediante el cual, al no poder surgir un acuerdo en torno a las partidas presupuestarias para los distintos sectores, la administración pública cesa todo servicio que no sea considera prioritario por ley (Brass, 2013).

${ }^{5}$ Esto convertía a los mexicanos en el grupo migrante más grande en el país. Compárese con la segunda mayoría compuesta por 2.1 millones de migrantes provenientes de China, Hong Kong y Taiwán (Herrara Lima, 2012)

${ }^{6}$ Reyneri (2001) señala que muchos migrantes presentan ciclos en los que regularizan su situación legal para luego perder este estado.
} 
mucho migrantes ingresan de manera regular pero que, al extender su estadía o desempeñar trabajos sin los permisos pertinentes, pierden dicha condición. A su vez, algunas investigaciones señalan que los migrantes propenden a regular su situación, ya sea por medios ilegales, como la compra o renta de documentación (Vasta, 2011) o el uso de programas especiales gubernamentales (Castles \& Miller, 2009).

Las corrientes migratorias no son un hecho histórico novedoso para los Estados Unidos, por ejemplo, gran parte de las corrientes migratorias modernas iniciaron en 1980, cuando los países más australes atravesaban dictaduras o enfrentaban un desastre natural (Herrera Lima, 2012). A los factores previamente señalados se le suman otros de carácter netamente económicos, por ejemplo, Viteri (2015) analiza la diferencia salarial del periodo 2002-2012 entre Estados Unidos y México, concluyendo que los ciclos de crisis económica afectan las decisiones de desplazamiento de los migrantes ${ }^{7}$.

Por otro lado, en la anterior administración del gobierno estadounidense, con el demócrata Barack Obama presidiendo desde el año 2009 hasta el 2017, no se llevaron cambios mayores en torno a las políticas migratorias. Velázquez Flores (2011) atribuye la permanencia del status quo a diferentes grupos de poder, señalando además que los ensayos para establecer políticas pro-migrantes ${ }^{8}$ respondían a intentos de captar el voto hispano $^{9}$.

A pesar de que, como ya se ha señaló, ante el atentado del 11 de septiembre del 2001 se produjo una marcada intensificación del control migratorio, la fortificación del aparato legislativo que facilitaba la deportación ya había dado inicio en 1988 con la ley antidrogas, pasándose de 4 felonías a 50 (Yrazir Barbosa \& Alarcon, 2015).

Tapia (2006) destaca el Programa Bracero ${ }^{10}$, llevado adelante entre los años 1942 y 1964 e impulsado por los efectos de la Segunda Guerra Mundial, como un hito que ejemplifica cómo los momentos de apertura y cierre ante la migración dependen de necesidades político-

\footnotetext{
${ }^{7}$ Se ha de señalar que esto a su vez fomenta la migración irregular; algunos sectores estadounidenses, ante las crisis económicas, tienden a contratar a migrantes sin permiso de trabajo puesto que suponen menores costos (Viteri, 2015).

8 Velázquez Flores (2011) se refiere a la promulgación del programa D.A.C.A. por parte de la administración Obama.

9 Considérese que desde el año 2000 los hispanohablantes son la primera minoría en Estados Unidos, a lo que se suma una creciente concientización por parte de dicha comunidad de su poder político, hecho que se vio reflejado en el aumento de votantes hispanos y las masivas marchas del año 2006 contra la criminalización de los inmigrantes (Morales, 2007).

10 El programa, cuyo nombre formal era el mexican farm labor program, fue un acuerdo entre Estados Unidos y México en el que se movilizó casi 5 millones de granjeros mexicanos para trabajar en los campos estadounidenses. No puede obviarse que dicho programa generó, como efecto no deseado, un aumento exponencial de migrantes irregulares; un hecho interesante era que ninguna de las dos naciones se encargaba de la situación e incluso se la abordaba con medidas cuasi cómicas: por ejemplo, las patrullas fronterizas llevaban a los migrantes irregulares hasta la frontera, los hacían pisar simbólicamente suelo mexicano y al volver eran admitidos legalmente (Durand, 2007).
} 
económicas, las cuales a su vez están condicionadas por factores como la opinión pública ${ }^{11}$ o grupos de poder. Además, se debe tomar en cuenta que el mencionado programa ya encontraba su germen en prácticas establecidas desde los años 1900.

Por último, si retrotraemos el análisis hasta los inicios de la historia norteamericana, se verá que la tendencia preeminentemente pragmática ante la otredad ${ }^{12}$ se presenta ya en su fundación. Kymlicka (1996) señala cómo en los Estados Unidos se llevó adelante una división territorial tal, diferente a otros países, en la cual se aisló y quitó representatividad, de manera intencional, a las minorías que conformaban la prehistoria de la nación.

\section{Conclusiones}

A lo largo del presente texto se han asentando diferentes posturas ante las preguntas iniciales. Es claro que las políticas migratorias estadounidenses atraviesan una suerte de aprovechamiento de las circunstancias, en el que los procesos de apertura y cierre no están directamente influidos por el partido gobernante. A modo de ejemplo, estadísticamente en los dos mandatos de Obama se sucedieron más deportaciones que los anteriores 30 años (Deparment of Homeland Security, 2014), comparativamente, en el primer año de presidencia de Trump se deportó menos, pero se sucedieron mayores arrestos y deportaciones de migrantes previamente residentes (US Inmigration and Custom Enforcement, 2018).

Por otro lado, las políticas que dificultan o directamente obstaculizan la migración no tienen nada de novedosas dentro de la historia estadounidense. A pesar de lo breve, se ha intentado señalar cómo, desde los momentos fundacionales hasta la actualidad, la relación con el migrante está sesgada por una tendencia que aprovecha de manera hábil las oportunidades de la circunstancia, sean motivos económicos -en tanto el migrante como mano de obra barata-, sea motivos políticos -en tanto potenciales votantes- o sean motivos más propios de la opinión pública -el migrante como depositario de sentimientos negativos en justificación de una situación adversa-. A su vez, no se puede dejar de señalar que esta labilidad anímica ante los migrantes tiene su correlato en el plano jurídico. De Cabo y Criado (2006) destacan cómo las legislaciones estadounidenses, en particular su constitución, cobran un carácter simbólico ${ }^{13}$ y que la jurisprudencia tiende a estar dominada por una ambivalencia más propia de la opinión pública que de un historial normativo aséptico.

\footnotetext{
11 Se utiliza el concepto para aludir a la pluralidad de opiniones e intereses dentro de un conjunto social y los particulares efectos que esto tiene sobre las decisiones políticas (Aguilar Villanueva, 2017).

12 Se utiliza el término dentro de las concepciones de Edward Said sobre la otredad cultural (Zapata Silva, 2008). ${ }^{13}$ La constitución, más allá de ser un documento, sería percibida como un legado de valores heredado por los padres fundadores, lo que a su vez le confiere una fuerte carga afectiva (De Cabo \& Criado, 2006).
} 
Por último, y a modo de cierre, es claro que ante semejante escenario la situación de los migrantes en suelo estadounidense se torna compleja, requiriendo de la organización y constante movilización por parte de los mismos para sostener sus derechos. Cierto es que Trump puso en práctica una serie de medidas que endurecieron la situación de los migrantes, pero más cierto es que esto es sólo parte de un ciclo que existe desde la fundación de su país.

\section{Referencias}

Adelman, H. y Taylor, L. (2015). Immigrant Children and Youth in the USA: Facilitating Equity of Opportunity at School. Education Sciences, 5(4), 323-344.

Aguilar Villanueva, L. F. (2017). Una reconstrucción del concepto de opinión pública. Revista mexicana de opinión pública, 23, 125-148.

Brass, C. T. (2013). Shutdown of the Federal Government: Causes, Processes, and Effects. Washington, DC: Congressional Research Service.

Castles, S. (2010). Migración irregular: Causas, tipos y dimensiones regionales. Migración y desarrollo, 7(15), 49-80.

Castles, S. y Miller, M. (2009). The Age of Migration: International Population Movements in the Modern World. Nueva York, United State of America: Palgrave Macmillan Higher Education.

De Cabo, A. \& Criado, M. (2006). How democratic is the american constitution? La actualidad constitucional en EE UU en 2005. Teoría y realidad constitucional, 18, 381-396.

Durand, J. (2007). El programa bracero (1942-1964). Un balance crítico. Migración y Desarrollo, 9(2), 27-43.

Herrera Lima, F. (2012). La migración a Estados Unidos: Una visión del primer decenio del siglo XXI. Norteamerica, 7(2), 171-197.

Kymlicka, W. (1996). Ciudadanía multicultural. Barcelona: Editorial Paidós.

Morales, W. (2007). La migración: un asunto de seguridad nacional en los Estados Unidos en el siglo XXI. México y la Cuenca del Pacifico, 10(28), 43-52. 
Passel, J. (2006). The Size and Characteristics of the Unauthorized Migrant Population in the U.S. Estimates Based on the March 2005 Current Population Survey. Recuperado de http://www.pewhispanic.org/files/reports/61.pdf

Tapia, C. E. (2006). Los programas de trabajadores temporales en la política migratoria estadunidense y la propuesta del presidente Bush. Norteamérica, 1(1), 247-258.

Tuirán, R. \& Avila, J. L. (2013). Migración calificada entre México-Estados Unidos: Desafíos y opciones de política. Migración y Desarrollo, 11(21), 43-63.

Vasta, E. (2011). Immigrants and the paper market: borrowing, renting and buying identities. Ethnic and Racial Studies, 34(2), 187-206.

Velazquez Flores, R. (2011). La política exterior de Estados Unidos hacia México bajo la administración de Barack Obama: cambios y continuidades. Norteamérica, 6(2), 85-113.

Viteri, J. (2015). Determinantes de la Migración México-Estados Unidos: estados expulsores y receptores, 2002-2012. Revista GeoPantanal, 18, 183-192.

Warren, R. (2016). US Undocumented Population Drops Below 11 Million in 2014, with Continued Declines in the Mexican Undocumented Population. Journal of Migration and Human Security, 4(1), 1-15.

Yrazir Barbosa, G. \& Alarcon, R. (2015). Las familias mexicanas con estatus inmigratorio mixto y la deportación masiva de Estados Unidos. REMHU, 23(45), 77-92.

Zapata Silva, C. (2008). Edwar said y la otredad cultural. Atenea (concepción), 498, 55-73.

\section{Documentos de trabajo}

Department of Homeland Security. (2014). Yearbook of immigration statistic. Washington DC, United States of America: Library of Congress.

International Organization for Migration. (2010). Irregular migration and mixed flows. Recuperado de http://publications.iom.int/system/files/pdf/wmr2010_irregular_migration_and_mix ed_flows.pdf 
López, M. H., Morin, R. \& Taylor, P. (2010). Illegal Immigration Backlash Worries, Divides Latinos. Recuperado de http://www.pewresearch.org/wpcontent/uploads/sites/5/reports/128.pdf

Reyneri, E. (2001). Migrants in irregular employment in the Mediterranean countries of the European Union. Recuperado de http://www.ilo.org/wcmsp5/groups/public/--ed_protect/---protrav/---migrant/documents/publication/wcms_201875.pdf

US Immigration and Custom Enforcement. (2018). Fiscal Year 2017 ICE Enforcement and Removal Operations Report. Recuperado de https://www.ice.gov/sites/default/files/documents/Report/2017/iceEndOfYearFY2017. pdf

Zaveri, M., Gates, G. \& Zraick, K. (2019). The Government Shutdown Was the Longest Ever. Here's the History. The New York Times. Recuperado de https://www.nytimes.com/interactive/2019/01/09/us/politics/longest-governmentshutdown.html 